\title{
Paradoxical reaction of tuberculosis masquerading as a gastric subepithelial tumor
}

Jinmo Kim', Kwangwoo Nam', Jooyeop Lee ${ }^{1}$, Jun-Ho Choi', and Won-Ae Lee ${ }^{2}$

Departments of ${ }^{1}$ Internal Medicine and ${ }^{2}$ Pathology, Dankook University Hospital, Dankook University College of Medicine, Cheonan, Korea
Received : August 26, 2021

Revised : August 31, 2021

Accepted: August 31, 2021

\section{Correspondence to}

Kwangwoo Nam, M.D.

Tel: +82-41-550-3092

Fax: +82-41-550-7050

E-mail: nambag1108@gmail.com https://orcid.org/0000-0003-

3720-9820
A 27-year-old man presented with severe upper abdominal pain for 3 weeks. It was aggravated after meal. He was diagnosed with tuberculous pleurisy 2 months ago, and receiving the standard 6-month anti-tuberculosis medications including isoniazid, rifampicin, ethambutol, and pyrazinamide. He had not taken nonsteroidal anti-inflammatory drugs or aspirin during the follow-up period and pain control was done using acetaminophen. Abdominopelvic computed tomography (CT) revealed a $3-\mathrm{cm}$ round gastric mass with central ulceration in the upper body, which were not prominent in the previous CT scan 2 months ago. Upper endoscopy revealed $3 \mathrm{~cm}$-sized subepithelial mass with an ill-defined central ulceration on the midbody (Fig. 1A). Endoscopic ultrasound (EUS) revealed a heterogenous hypoechoic mass and central hyperechoic foci (Fig. 1B).
Forceps biopsy and fine needle aspiration biopsy (FNAB) were performed without complications. The histologic diagnosis was chronic granulomatous inflammation with ulceration, consistent with tuberculosis (Fig. 2A). Although tissue culture and polymerase chain reaction was negative for tuberculosis, the final diagnosis was a paradoxical reaction of tuberculosis. Thus he continued to receive anti-tuberculosis medications as scheduled. Then upper gastrointestinal symptom gradually improved without additional complication. Follow-up endoscopy and CT scan after 3 months later revealed complete resolution of the gastric mass (Fig. 2B). Informed consent was obtained from the patient.

Extrapulmonary paradoxical reaction of tuberculosis in the stomach is uncommon. Gastric tuberculosis may appear in the form of ulcer or subepithelial tumor.
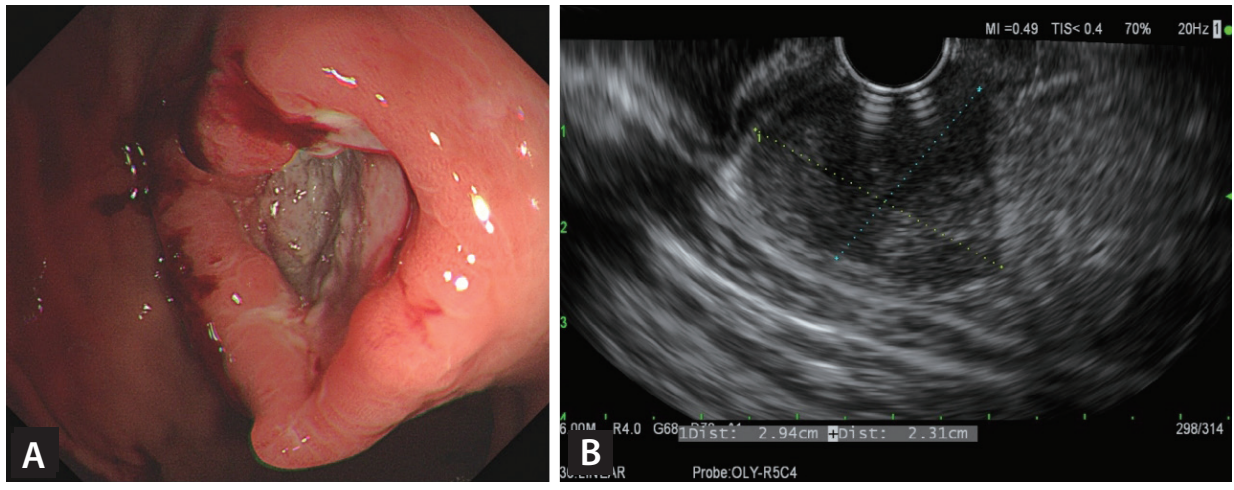

Figure 1. (A) Upper endoscopy images showed a 3-cm sized gastric mass with irregular-margined ulcer. (B) Endoscopic ultrasound imaged showed a heterogenous hypoechoic mass with central hyperechoic foci. 


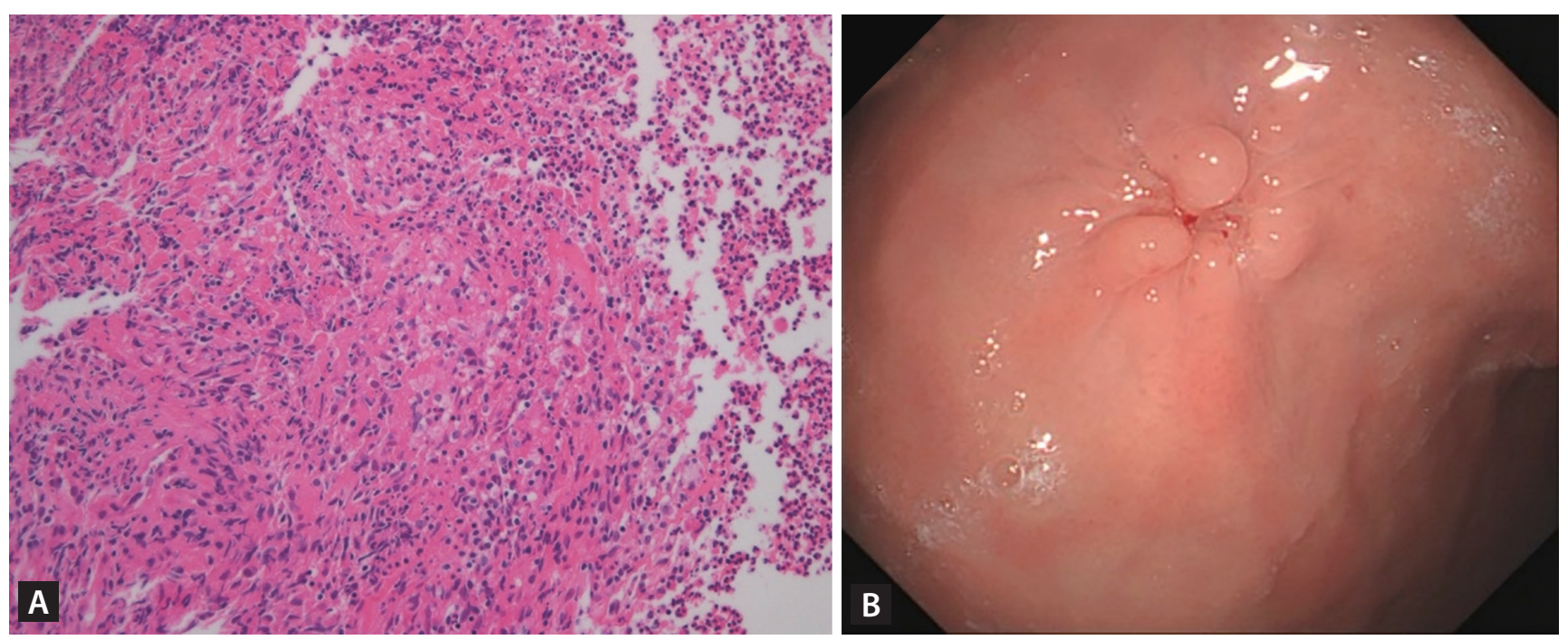

Figure 2. (A) Histopathologic finding revealed granulomatous inflammation which was consistent with tuberculosis (H\&E, x400). (B) Follow-up upper endoscopy images showed complete resolution of gastric mass-like lesion.

It reportedly occurs within 3 months after initiation of anti-tuberculous medications. The mainstay of treatment is to maintain the current treatment. However, treatment failure and drug resistance should be excluded before the decision. In this situation, EUS-FNAB in addition to forceps biopsy can be a feasible option to confirm the diagnosis.

\section{Conflict of interest}

No potential conflict of interest relevant to this article was reported. 